\title{
Os cuidados de Enfermagem na Síndrome de Stevens Johnson /Necrólise Epidérmica Tóxica: Um Relato de Caso.
}

\section{Nursing care in the Stevens-Johnson Syndrome and Toxic Epidermal Necrolysis:A Case Report.}

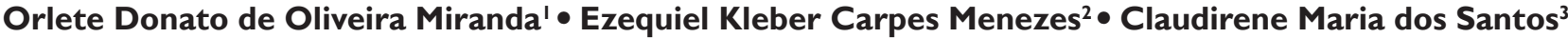 \\ - Luiz Carlos Porcello Marrone ${ }^{4}$ Claudemir Monteiro de Barros ${ }^{5}$
}

\begin{abstract}
RESUMO
A síndrome se Síndrome de Steven Johnson (SSJ)/Necrólise Epidérmica Tóxica (NET) apresenta-se como uma afeção inflamatória aguda, caracterizada pelas manifestações cutâneas, que podem afetar outros sistemas dependendo do estágio. Há dois objetivos específicos: Descrever o caso de um paciente pediátrico com SSJ/NET em uma Unidade de Terapia Intensiva Pediátrica de uma organização pública de saúde no interior do estado de Rondônia, contrastando o atendimento local com a literatura vigente, além de sugerir propostas de diagnósticos e intervenções de enfermagem. Metodologia: $O$ presente estudo trata-se de um relato de caso de um paciente pediátrico acometido pela SSJ e NET a partir dos prontuários fornecidos pela genitora do paciente, no momento da alta hospitalar. Os dados foram armazenados em um banco de dados eletrônico e estudados a partir do método descritivo de análise documental. Como resultados e discussão do caso discutido foram evidenciados quinze diagnósticos de enfermagem e as intervenções de enfermagem correspondentes. Conclui-se que a imunoglobulinoterapia mostrou-se eficaz no tratamento e que a implementação da assistência de enfermagem pode ser considerada parte essencial no tratamento da SSJ/NET.
\end{abstract}

Palavras-chave: Síndrome de Stevens-Johnson, Imunoglobulinas Intravenosas, Diagnóstico de Enfermagem; Intervenções de Enfermagem.

\begin{abstract}
The Stevens Johnson Syndrome (SSJ) / Toxic Epidermal Necrolysis (NET) is presented as an acute inflammatory condition typically characterized by cutaneous manifestations, which may affect other systems depending on the stage. There are two specific objectives:To describe the case of a pediatric patient with SSJ / NET in a Pediatric Intensive Care Unit of a public health organization in the interior of the state of Rondônia, contrasting the local care with the current literature, and suggesting proposals for nursing diagnoses and interventions. Methodology: The present study obtain a case report of a pediatric sick person affected by SSJ and NET from the records provided by the patient's parent at the time of hospital discharge. Data were accumulated in an electronic database and studied using the descriptive method of document analysis. As results and discussion of the case discussed were evidenced fifteen nursing diagnoses and the corresponding nursing interventions. It is concluded that immunoglobulin therapy was effective in the care and that the implementation of nursing care can be considered an essential part in the treatment of SSJ / NET.
\end{abstract}

KeyWords: Stevens-Johnson Syndrome; Immunoglobulins, Intravenous; Nursing Diagnosis; nursing interventions

I Graduada em Enfermagem pelo Centro Universitário Luterano de Ji-Paraná. Mestranda pela Universidade Luterana do Brasil e docente do curso de Enfermagem da Faculdade pan-americana de Ji-paraná (UNIJIPA).Email: orletedonato@gmail.com

2 Graduando em Enfermagem pelo Centro Universitário São Lucas. Email: ezequielkleber@gmail.com

3 Graduada em Enfermagem pelo Centro Universitário São Lucas. Email: claudirenemaria736@gmail.com

4 Graduado em medicina pela Universidade Luterana do Brasil (ULBRA), Doutor em Neurociências (Medicina) pela Pontificia Universidade Católica do Rio Grande do Sul. Professor de Neurologia da Faculdade de Medicina ULBRA e do Programa de Pós Graduação em Promoção da Saúde da ULBRA/Canoas. Pesquisador do Instituto do Cérebro do Rio Grande do Sul (PUCRS). Email:

5 Graduado em Medicina pela FACIMED - Faculdade de Ciências Biomédicas de Cacoal. Residência Médica em Pediatria pelo Hospital Regional de Cacoal (HRC) / Secretaria Estadual de Saúde de Rondônia (SESAU/R0). Membro titulado (TEP) da Sociedade Brasileira de Pediatria (SBP). Membro sócio da AMIB (Associaçao de Medicina Intensiva Pediatrica). Coordenador interino da UTI Pediátrica do HRC/SESAU/RO e visitador diarista (estatutário). 


\section{INTRODUÇÃO}

A Síndrome de Steven Johnson (SSJ) foi descrita pela primeira vez em 1922, por Stevens e Johnson, ao relatarem dois casos de erupções cutâneas generalizadas, febre contínua, mucosa oral inflamada e conjuntivite purulenta grave ${ }^{(1)}$.A síndrome se apresenta por uma afecção inflamatória aguda, febril e autolimitada, com duração aproximada de quinze a trinta dias, caracterizada pela apoptose dos queratinócitos, afetando o tecido tegumentar e a mucosa ${ }^{(2)}$.

Estudo transversal investigou a epidemiologia Síndrome de Steven Johnson (SSJ)/Necrólise Epidérmica Tóxica (NET) nos Estados Unidos entre os anos de 2009 e 20 I2, ocasião em que os autores observaram uma taxa de incidência global de 12.7 casos/milhões de adultos ${ }^{(3)}$. Levantamento epidemiológico realizado na Coreia (cobertura de $97 \%$ da população) evidenciou uma taxa de incidência de 5,9 casos SSJ/NET/milhões de pessoas entre os anos de 2010 e $2013^{(4)}$. No Brasil os dados relacionados à SSJ e NET são escassos, no entanto, existe uma estimativa de I,2 - 6 casos/milhões de habitantes e 0,4 - I,2 casos/ milhão de habitantes por ano, respectivamente ${ }^{(5)}$.

A faixa etária com maior prevalência são de crianças com idade entre I e 10 anos $(7,63-8,97$ casos por milhões de pessoas) e em pacientes com idade igual ou superior a 80 anos $(8,75$ casos por milhões de pessoas) ${ }^{(6)}$. Essa síndrome, embora apresente uma taxa de mortalidade menor que $5 \%$, quando em SSJ, pode aumentar para cerca de 30 a $50 \%$ nos pacientes com NET. Nesses casos as mortes acontecem ainda na fase aguda da desordem ${ }^{(7)}$.

A fase aguda da doença tem início súbito e ocorre nos primeiros 8 a 12 dias, quando apresenta como principais sintomas febre de $39-40^{\circ} \mathrm{C}$, algia, mal-estar, cefaleia e odinofagia. Após esse estágio, ocorre o agravamento dos sintomas, podendo evoluir para pulso filiforme e acelerado, taquipneia, prostração, artralgias, mialgias e uma propagação rápida de petéquias e/ou vesículas bolhosas, com inflamações por todo corpo, além de orifícios, como boca, nariz, conjuntiva, uretra, vagina e ânus ${ }^{(5,8)}$.

É importante destacar que, além das manifestações cutâneas, ocorrem também manifestações no trato gastrointestinal, tais como hiporexia, diarreia e hemorragias. No sistema respiratório podem ser observados, por meio de radiografia, derrame pleural e edema agudo de pulmão, bem como dispneia e, em muitos casos, o desenvolvimento de pneumonia ${ }^{(5,7)}$.

Em razão da variabilidade de sinais e sintomas clínicos, Bastuji-Garin et al propuseram, em 1993, uma classificação para SSJ ${ }^{(9)}$. Segundo essa classificação, de acordo com a porcentagem do acometimento epidérmico pelas lesões, difere-se apenas na superfície corporal afetada, definida pelo destacamento da epiderme ou sinal de Nikol- sky positivo (separação epidérmica induzida por pequena pressão lateral da superfície cutânea) $)^{(7,8)}$. Quando $<10 \%$, denomina-se SSJ; entre 10 e 30\%, define-se sobreposição SSJ/NET (overlap); e quando $>30 \%$, de acordo com Lyell, Necrólise Epidérmica Tóxica (NET), que se caracteriza pela presença de máculas purpúricas disseminadas e necrose isolada da camada epidérmica pelo destacamento da epiderme ${ }^{(1,10)}$.

Também foi estabelecido o escore de gravidade da doença (SCORTEN), para prever o risco de mortalidade. Os sete fatores a seguir foram considerados de alto risco, com cada um equivalente a um ponto: idade $>40$ anos, presença de malignidade, frequência cardíaca $>120$ bpm, nível de ureia sérica $>10 \mathrm{mmol} / \mathrm{L}(>27 \mathrm{mg} / \mathrm{dl})$, porcentagem de descolamento epidérmico $>10 \%$ da área de superfície coporal, nível sérico de glicose $>14 \mathrm{mmol} / \mathrm{L}$ (> $250 \mathrm{mg} / \mathrm{dl}$ ) e bicarbonato sérico nível $<20 \mathrm{mEq} / \mathrm{L}$. Um escore maior significa um risco maior de mortalidade. Sendo, de acordo com o sistema de classificação SCORTEN: 0-I ponto, 3\%; 2 pontos, $12 \%$; 3 pontos, $35 \%$; 4 pontos, $58 \%$; e 5 pontos ou mais, $>90 \%{ }^{(8,9,10)}$.

A fisiopatologia, embora não seja totalmente conhecida, sabe-se que ocorre pela combinação da estrutura química da droga e fatores genéticos. Essa relação ocorre de duas formas: a primeira é por meio de mecanismos farmacológicos, em que ocorre uma alteração de enzimas metabolizadoras, das proteínas transportadoras e no próprio alvo farmacológico; a segunda é a ocorrência de modificações do sistema imunitário, envolvendo a molécula HLA(II).

Um problema observado em relação às formas de conduta é que, devido à raridade desses distúrbios, não existem grandes estudos controlados randomizados. Essa condição faz do tratamento um desafio, pois, embora as condutas de descontinuação do agente agressor e manutenção do equilíbrio hemodinâmico e prevenção de complicações potencialmente fatais (cuidados de suporte) sejam primordiais, não existe uma regra para o tratamento sobre o uso ideal de agentes imunomoduladores sistêmicos adjuntos. Essa ausência de diretriz permite a utilização de diversas formas de tratamento. Dentre os relatados, a forma mais comum é a Imunoglobulina (IVIG) ${ }^{(12)}$.

Outra lacuna que deve ser formentada é o fato de não ser encontrado na literatura de enfermagem acerca desse assunto. E diante da gravidade da SSJ/NET e da não padronização do tratamento tornou-se relevante a realização deste estudo, utilizando o processo de enfermagem (PE).

O PE é um caminho metodológico, sistematizado e dinâmico para a assistência de enfermagem ao indivíduo, família ou comunidade. A mesma é composta por etapas inter-relacionadas que se iniciam com a identificação das necessidades apresentadas pelo paciente, seguindo logo 
após a elaboração dos diagnósticos, geralmente utilizando o North American Nursing Diagnosis Association Internacional (NANDA) $)^{(13)}$. A partir disso, são realizados o planejamento das intervenções, a partir da Classificação das Intervenções de Enfermagem (NIC) ${ }^{(14)}$ sendo implementados e avaliados os resultados.

Então, frente à singularidade da patologia e levando em consideração a importância de aprofundamento no tema, esta pesquisa possui dois objetivos específicos. São eles: descrever o caso de um paciente pediátrico com SSJ/NET em uma Unidade de Terapia Intensiva Pediátrica de uma organização pública de saúde no interior do Estado de Rondônia, contrastando o atendimento realizado no local com a literatura vigente, além de sugerir propostas de diagnósticos e intervenções de enfermagem.

\section{METODOLOGIA}

Trata-se de um estudo qualitativo, exploratório, do tipo relato de caso de análise retrospectiva, realizado por meio de coletas do prontuário do paciente, apresentando os aspectos sobre as manifestações clínicas, tratamento e cuidados de enfermagem em um paciente pediátrico com SSJ, no internado em um hospital do interior do estado de Rondônia, no ano de 2017.

Este faz parte de um projeto de pesquisa em enfermagem, aprovado pelo Comitê de Ética em Pesquisa (CEP) do Centro Universitário Luterano de Ji-Paraná (CEULJI-ULBRA), pelo Parecer $n^{\circ}$ 2.163.475 e CAAE 7080 I 617.3 .0000 .5297 . Os prontuários analisados foram fornecidos pela genitora do paciente, no momento da alta hospitalar, seguindo os princípios éticos e legais, estabelecidos pela portaria 466/ 2012 garantindo, também, que todas as informações fornecidas, bem como o nome e dados de identificação, permanecessem em total sigilo.

A escolha do paciente se deu a partir do interesse em contribuir para disseminação do conhecimento, por meio do levantamento de dados com vistas a construir os diagnósticos e as intervenções de enfermagem em um paciente com SSJ/NET, além de contrastar os sinais e sintomas clínicos mais citados na literatura com os observados no paciente deste caso.

Foi constituída uma planilha eletrônica para o armazenamento dos dados, por meio do programa Microsoft Office Excel 2016. Onde todas as informações obtidas a partir dos históricos dos 3 (três) volumes do prontuário, fornecidos pela genitora, dos três hospitais onde o paciente esteve internado foram preenchidas. A partir desses dados, foi possível analisar sua evolução no período de 27 de setembro (data em que fora registrada sua entrada no hospital municipal de Ouro Preto D'Oeste) a I4 de outubro e 2017 (data da alta do hospital Regional de Cacoal).

Foram retiradas informações das prescrições médicas e de enfermagem, tais como: história clínica pregressa, an- tecedentes familiares, história da doença atual, evolução clínica e de enfermagem, diagnósticos médico e de enfermagem e descrição dos cuidados e condutas médicas e de enfermagem realizadas durante todo o período de hospitalização. Vale ressaltar que na instituição não há a implementação da sistematização de Enfermagem, no entanto para que houvesse maior compreensão do assunto, os autores da presente pesquisa utilizou tal metodologia.

Todos os dados foram armazenados em um banco de dados eletrônico, seguindo os procedimentos de análise do método descritivo de análise documental. A primeira etapa da análise de dados almejou a descrição do caso elencando as principais necessidades identificados no paciente e, após esse estágio, os autores da presente pesquisa apresentaram os principais diagnósticos de enfermagem, fundamentados na North American Nursing Diagnosis Association (NANDA) ${ }^{(13)}$, que representaram a base para a escolha das intervenções e resultados terapêuticos. Na constituição das propostas de intervenções de enfermagem, por apresentar muitas possibilidades, utilizou-se a Classificação da Nursing Interventions Classification (NIC) ${ }^{(14)}$. Após toda essa estruturação, os achados foram discutidos com base na literatura pertinente.

\section{RELATO DE CASO}

O paciente, do sexo masculino, pardo, 13 anos, procedente de um município do interior de Rondônia, acompanhado por sua progenitora, fora admitido no dia 02.10.2017 em uma Unidade Intensiva Pediátrica (UTI $-P)$, proveniente de um hospital de urgência emergência, onde recebeu o primeiro atendimento, com hipótese diagnóstica de Necrólise Epidérmica Tóxica (síndrome de Lyell)(?).

Durante a anamnese, a genitora relatou o surgimento de lesões 5 dias antes do dia da internação, cursando com quadro de febre, dor abdominal e cefaleia, sendo automedicado com 20 gotas de dipirona. No dia seguinte ao aparecimento das lesões, queixou-se dos mesmos sintomas e eclosão de lesões bolhosas em tórax e prurido. A partir desse quadro, a genitora procurou atendimento médico no hospital do município de origem, onde foi prescrito tratamento sintomático, dipirona 25 gotas de 4/4 horas. Após 2 dias, o adolescente evoluiu com queda do estado geral e presença de lesões bolhosas e pruriginosas difusas, com áreas hipercrômicas e descamação dérmica por todo o corpo, principalmente tronco e dorso, sendo, então, encaminhado para uma unidade de urgência e emergência estadual, referência de atendimentos na região central do estado de Rondônia.

Após o procedimento de transferência, o paciente fora admitido na unidade de urgência e emergência em 01.10.2017, queixando-se de dificuldade para ingerir alimentos, com quadro de taquicardia, taquipneia, febre, pele 
e mucosas hipocoradas, com presença de lesões bolhosas difusas, com áreas hipercrômicas e descamação dérmica em tórax e abdômen anterior e toda região dorsal, cavidade oral com lesões crostosas e dolorosas. Foi iniciada antibioticoterapia, com cefriaxona $2 \mathrm{~g} /$ dia e vancomicina $60 \mathrm{mg} /$ $\mathrm{kg} / \mathrm{dia}$ e, em razão de quadro de septicemia, procedeu-se solicitação de vaga em terapia intensiva.

À admissão na UTI pediátrica, o paciente se encontrava consciente, orientado, contactuante, mas sem verbalizar devido às lesões na mucosa oral, deambulando com dificuldade, necessitando auxílio em virtude das vesículas bolhosas pelo corpo, normotenso, taquipneico, taquisfígmico, afebril, acianótico, edema ++/4+ em face e membros superiores e inferiores, com integridade da pele (cutâneo mucosa) comprometida: lesões, tipo queimadura de $2^{\circ}$ grau, espalhadas pelo corpo uniformemente, com secreção piosanguinolenta, espessa e fétida. Presença de lesões em mucosas oral. Tórax: ausculta cardíaca com presença de sopro, ausculta pulmonar sem alterações. Pulso cheio e rítmico, abdômen plano, com ruídos hidroaéreos presentes. Presença de lesões em genitália. Eliminações vesicais presentes, via cateter vesical de demora. Evacuação ausente no momento da internação.

$\mathrm{Na}$ Unidade Intensiva Pediátrica foi iniciado suporte nutricional enteral, mantida a antibioticoterapia e curativo diário com cobertura de vaselina sólida e ácidos graxos essenciais (AGE), além do desbridamento cutâneo. Devido ao ressecamento ocular e lesões próximas à região ocular, foi solicitado avaliação com oftalmologista, que prescreveu colírio trisorb 2/2 horas. Mantida a antibioticoterapia.

No 4 DI (dias de internação), na avaliação psicológica foi observado paciente receptivo, colaborativo e calmo. Queixava-se apenas no banho e na troca de curativo e manifestava baixa autoestima em razão das diversas lesões no corpo, devido a isso, o corpo de enfermagem realizou medidas de controle de dor e analgesia conforme prescrição médica. Bem como encaminhamento para psicólogo onde também houve a participação dos progenitores. Mãe e pai condizentes, apresentando-se bastante emotivos e preocupados com o caso do filho.

No $5 \mathrm{DI}$, diante da pouca melhora clínica e tratando-se de NET, optou-se por administrar Imunoglobulina humana $\mathrm{EV} \mathrm{Ig} / \mathrm{kg} / \mathrm{dia}$. Embora $\circ$ adolescente relatasse fortes dores, sendo necessária a administração de medicamentos na realização dos curativos, apresentou melhora significativa após a primeira semana de internação na UTI-P evoluindo com diminuição das lesões em mucosa oral, reestabelecendo a dieta via oral; redução das lesões bolhosas, com o aparecimento de crostas e reepitelização da pele, além de ausência de febre.

Devido à melhora clínica, no $9^{\circ}$ dia de internação em Unidade Terapia Intensiva Pediátrica, optou-se por transferência para enfermaria. $\mathrm{Na}$ admissão na enfermaria, o me- nor apresentava-se ativo; reativo; eupneico; com mucosa oral íntegra; ausculta cardíaca sem alterações; ausculta pulmonar sem alterações, com múrmuros vesiculares presentes, sem ruídos adventícios; abdômen indolor à palpação, com ruídos hidroaéreos presentes; em uso de cefriaxona, $100 \mathrm{mg} / \mathrm{kg} /$ dia, iniciado em 01.10 .2017 (reduzido para 2g/ dia em 05. 10.2017 ); e de vancomicina $60 \mathrm{mg} / \mathrm{kg} / \mathrm{dia}$, iniciado em 02.10.2017 (reduzido para $40 \mathrm{mg} / \mathrm{kg} / \mathrm{dia}$ ).

Vale ressaltar que as informações adquiridas foram recolhidas exclusivamente nos prontuários do paciente que foi disponibilizado pela genitora. Isso explica o fato de os cuidados prestados, pela equipe de enfermagem, à internação na UTI-ped não terem sido levantados. Como também, pela SSJ/NET ser uma enfermidade rara, não existem protocolos de cuidados para esse tipo de paciente nos hospitais em que foi atendido, por isso a assistência realizada foi baseado nas necessidades do paciente.

Face ao ocorrido, e sabendo que há uma lacuna na literatura de enfermagem sobre este tema, tornou-se importante o levantamento dos diagnósticos de enfermagem pelos autores. Tendo por certo que o Processo de Enfermagem é a melhor opção para uma assistência de enfermagem de qualidade ao indivíduo, família ou comunidade, devendo ser operacionalizada em etapas inter -relacionadas a partir da identificação dos problemas de enfermagem, da formação de diagnóstico, do planejamento, implementação e avaliação ${ }^{(15)}$, o presente artigo selecionou 15 diagnósticos e os respectivos cuidados para cada um deles.

A presença de lesões bolhosas disseminadas secundárias à reação de hipersensibilidade, principalmente na região do dorso e do tronco, estão relacionadas à grande maioria dos diagnósticos de enfermagem. $O$ diagnóstico de integridade da pele prejudicada se relacionou com diversos outros, pois em razão do rompimento das camadas da pele, observou-se a mobilidade física prejudicada, dor aguda durante a realização da troca de curativo, risco de desequilíbrio eletrolítico, risco de volume deficiente de líquidos, assim como déficit no autocuidado para vestir-se.

Além da pele, as mucosas também foram afetadas, dentre elas a oral e a ocular. $\mathrm{Na}$ oral, os diagnósticos de enfermagem sugeridos foram a deglutição prejudicada, a integridade da mucosa oral prejudicada e a comunicação verbal prejudicada, evidenciados pelo comprometimento nutricional e fonético. $O$ paciente fez uso de dispositivo de alimentação enteral (Sonda Nasoenteral) que, embora seja uma forma adequada e eficiente para repor as necessidades calóricas do indivíduo, sua utilização leva a uma diminuição da quantidade de alimentos ingeridos, possíveis prejuízos na fala, bem como eventos decorrentes de erros por parte dos profissionais na inserção e/ou manutenção ${ }^{(16)}$. 
TABELA 1 - Diagnósticos de enfermagem baseados na NANDA (2018)(13) Cacoal, RO, Brasil, 2017.

\begin{tabular}{|c|c|c|}
\hline DIAGNÓSTICO DE ENFERMAGEM & FATOR RELACIONADO & CARACTERÍSTICAS DEFINIDORAS \\
\hline INTEGRIDADE DA PELE PREJUDICADA & $\begin{array}{l}\text { Secundária à reação de hipersensibilidade } \\
\text { à Dipirona. }\end{array}$ & Surgimento de lesões bolhosas. \\
\hline MOBILIDADE FÍSICA PREJUDICADA & $\begin{array}{l}\text { Presença de lesões bolhosas por todo o } \\
\text { corpo. }\end{array}$ & Dificuldade para mudar de decúbito. \\
\hline DOR AGUDA & Exposição de receptores nervosos. & $\begin{array}{l}\text { Relato verbal de dor durante o } \\
\text { prosseguimento da técnica de troca de } \\
\text { curativo e mobilização. }\end{array}$ \\
\hline $\begin{array}{l}\text { DÉFICIT NO AUTOCUIDADO PARA } \\
\text { VESTIR-SE }\end{array}$ & Dor & $\begin{array}{l}\text { Capacidade prejudicada de colocar roupas } \\
\text { na parte do tórax. }\end{array}$ \\
\hline DEGLUTIÇÃO PREJUDICADA & Presença de lesões na mucosa oral. & Recusa da ingestão e relato de dor \\
\hline $\begin{array}{l}\text { INTEGRIDADE DA MUCOSA ORAL } \\
\text { PREJUDICADA }\end{array}$ & $\begin{array}{l}\text { Secundária à reação de hipersensibilidade } \\
\text { à Dipirona. }\end{array}$ & $\begin{array}{l}\text { Lesões orais, hiperemia, desconforto oral e } \\
\text { disfasia. }\end{array}$ \\
\hline COMUNICAÇÃO VERBAL PREJUDICADA & $\begin{array}{l}\text { Utilização de sonda nasoenteral e presença } \\
\text { de lesões orais. }\end{array}$ & Dificuldade para falar. \\
\hline RISCO DE RESSECAMENTO OCULAR & Perda excessiva de líquidos pela pele. & \\
\hline RISCO DE LESÃO NA CÓRNEA & Reação de hipersensibilidade. & \\
\hline RISCO DE INFECÇÃO & $\begin{array}{l}\text { Pele rompida por lesões bolhosas. } \\
\text { Descolamento epidérmico. Uso de } \\
\text { dispositivos invasivos: cateter venoso } \\
\text { central e sonda vesical. }\end{array}$ & \\
\hline TERMORREGULAÇÃO INEFICAZ & Secundária à NET. & $\begin{array}{l}\text { Aumento de temperatura corporal acima dos } \\
\text { parâmetros normais. }\end{array}$ \\
\hline BAIXAAUTOESTIMA SITUACIONAL & $\begin{array}{l}\text { Alteração da imagem corporal devido às } \\
\text { lesões. }\end{array}$ & $\begin{array}{l}\text { Verbalizações autonegativas que refletem } \\
\text { uma visão alterada do próprio corpo na } \\
\text { aparência, estrutura ou função. }\end{array}$ \\
\hline VOLUME EXCESSIVO DE LÍQUIDOS & Hipersensibilidade medicamentosa. & $\begin{array}{l}\text { Edema }++/ 4+\text { em face e membros } \\
\text { superiores e inferiores. }\end{array}$ \\
\hline $\begin{array}{l}\text { RISCO DE DESEQUILÍBRIO } \\
\text { ELETROLITICO }\end{array}$ & $\begin{array}{l}\text { Mecanismo de regulação comprometido } \\
\text { (perda das camadas da pele). }\end{array}$ & \\
\hline $\begin{array}{l}\text { RISCO DE VOLUME DEFICIENTE DE } \\
\text { LÍQUIDOS }\end{array}$ & $\begin{array}{l}\text { Perda excessiva de líquido por vias normais } \\
\text { (lesões). }\end{array}$ & \\
\hline
\end{tabular}

Fonte: Dados do estudo, Cacoal, 2017.

Na mucosa ocular, em consequência da cicatrização tecidual que pode levar ao surgimento de simbléfaro ou anquilobléfaro e, tardiamente, de entrópio, triquíase, conjuntivalização e/ou ceratinização corneana, lagoftalmo e olho seco; e a ceratoconjutivite que, por sua vez, pode afetar a visão e retrair a conjuntiva, causar cicatrização e lesão da córnea, sepse e até mesmo a morte ${ }^{(2)}$. Os diagnósticos de enfermagem associados foram: risco de ressecamento ocular e risco de lesão na córnea (17).

A presença de quadro febril se relacionou ao diagnóstico de enfermagem de hipertermia. Tal diagnóstico se relaciona ao risco de infecção, que se manteve durante toda a fase de internação do paciente devido à contínua exposição, seja pelas próprias lesões, seja pelos procedimentos invasivos.

Por fim, o paciente e os familiares apresentaram problemas emocionais relacionados a este incidente. Foram levantados os diagnósticos de enfermagem de baixa autoestima situacional, em razão da preocupação do paciente em relação às marcas deixadas pelas lesões e com o receio de uma possível recidiva. Tais sinais foram observados durante todo o processo hospitalar e se mantiveram mesmo na alta.

Com relação aos cuidados de enfermagem, estes devem ser direcionados a partir da identificação dos problemas e diagnósticos de enfermagem. Baseado nesses achados, as principais intervenções levantadas para o paciente em questão foram: 
TABELA 2 - Diagnósticos e Intervenções de Enfermagem apreendidas pelos autores fundamentados na NANDA e na NIC, identificados com frequência no relato de caso. Cacoal, RO, Brasil, 2017.

\begin{tabular}{|c|c|}
\hline $\begin{array}{l}\text { Diagnóstico de Enfermagem }{ }^{(13)} \\
\text { (NANDA) }\end{array}$ & $\begin{array}{l}\text { Intervenções de Enfermagem }{ }^{(14)} \\
\text { (NIC) }\end{array}$ \\
\hline Integridade da Pele Prejudicada & $\begin{array}{l}\text {-Realizar curativo com a medicação tópica, adequada (se dor, aplicar analgesia); } \\
\text {-Observar sinais e sintomas de infecção; } \\
\text {-Observar e manter cuidados com áreas de pressão (Hidratar a pele, quando necessário) } \\
\text { - Realizar curativo na área afetada; e } \\
\text { - Observar alterações na pele. }\end{array}$ \\
\hline Mobilidade Física Prejudicada & $\begin{array}{l}\text { - Determinar a capacidade atual do paciente em transferir-se; } \\
\text { - Selecionar a técnica de transferência adequada ao paciente; } \\
\text { - Orientar o paciente sobre todas as técnicas apropriadas, almejando atingir o seu maior nível de } \\
\text { independência; } \\
\text { - Identificar métodos de prevenção de lesão durante as transferências; e } \\
\text { - Avaliar o paciente ao término da transferência quanto ao alinhamento corporal correto, a não oclusão } \\
\text { de sondas e drenos, roupas de cama com rugas e dobras, pele desnecessariamente exposta, nível } \\
\text { de conforto adequado para o paciente, laterais da cama levantadas e campainha ao alcance da mão. }\end{array}$ \\
\hline Dor Aguda & $\begin{array}{l}\text { - Realizar avaliação da dor com relação a local, características, o início/duração, a frequência, a } \\
\text { intensidade ou a gravidade da dor e os fatores agravantes; } \\
\text { - Observar indicadores não verbais de desconforto. Instituir e modificar medidas de controle da } \\
\text { dor com base na resposta do paciente; } \\
\text { - Investigar com o paciente os fatores que aliviam/pioram a dor; } \\
\text { - Assegurar analgesia pré-tratamento e/ou estratégias não farmacológicas antes de procedimentos } \\
\text { dolorosos; } \\
\text { - Fornecer ao paciente cuidados precisos de analgésicos prescritos; } \\
\text { - Selecionar e implementar uma combinação de medidas (farmacológicas, não farmacológicas, } \\
\text { interpessoais) para auxiliar no alívio da dor, quando adequado; } \\
\text { - Aplicar quente /frio quando apropriado; e } \\
\text { - Realizar controle da eficácia das medidas de controle da dor por meio de um levantamento } \\
\text { constante da experiência de dor. }\end{array}$ \\
\hline Déficit no autocuidado para vestir-se & $\begin{array}{l}\text { - Oferecer a roupa de modo a facilitar o acesso a elas (p.ex., junto ao leito); } \\
\text { - Manter a privacidade enquanto o paciente se veste; } \\
\text { - Incentivar e reforçar as tentativas de vestir-se sozinho; } \\
\text { - Identificar áreas em que o paciente precisa de auxílio para vestir-se; } \\
\text { - Monitorar a capacidade do paciente para vestir-se; } \\
\text { - Vestir o paciente após realização da higiene pessoal; } \\
\text { - Estimular a participação na escolha das roupas; } \\
\text { - Vestir primeiro a extremidade afetada, conforme apropriado; } \\
\text { - Vestir roupas que não limitem os movimentos do corpo, conforme apropriado; e } \\
\text { - Auxiliar até que o paciente esteja totalmente capacitado a assumir a responsabilidade pelo ato de vestir-se. }\end{array}$ \\
\hline $\begin{array}{l}\text { Risco de Ressecamento Ocular } \\
\text { Risco de Lesão na Córnea }\end{array}$ & $\begin{array}{l}\text { - Monitorar o reflexo da córnea; } \\
\text { - Orientar o paciente a não tocar nos olhos; } \\
\text { - Monitorar a ocorrência de vermelhidão, exsudato ou úlcera; } \\
\text { - Aplicar gotas lubrificantes, conforme apropriado; e } \\
\text { - Aplicar compressas úmidas, conforme apropriado. }\end{array}$ \\
\hline
\end{tabular}


Termorregulação ineficaz

Risco de infecção

- Monitorar a temperatura com a frequência adequada;

- Monitorar a cor e a temperatura da pele;

- Monitorar a pressão sanguínea, pulso e respiração, conforme apropriado;

- Monitorar a diminuição do nível de consciência;

- Monitorar a ingestão e eliminação;

- Monitorar anormalidades eletrolíticas;

- Administrar medicação para tratamento da causa da febre, conforme apropriado;

- Cobrir o paciente somente com lençol, conforme apropriado;

- Administrar banho morno de esponja, conforme apropriado;

- Estimular ingestão maior de líquidos orais, conforme apropriado;

- Aplicar à virilha e às axilas bolsa de gelo coberta com toalha;

- Aumentar a circulação de ar usando um ventilador; e

- Monitorar a temperatura atentamente para prevenir hipotermia induzida pelo tratamento.

- Instituir precauções de isolamento, conforme apropriado;

- Assegurar o manuseio asséptico de todas as linhas endovenosas;

- Assegurar o emprego da técnica adequada no cuidado de feridas;

- Estimular a ingestão hídrica, conforme apropriado;

- Estimular o repouso;

- Orientar o paciente e a família sobre os sinais e sintomas de infecção e sobre o momento de relatá-los ao profissional de saúde; e

- Ensinar ao paciente e familiares como evitar infecções.

- Monitorar a frequência de verbalizações autonegativas;

- Investigar as razões da autocrítica e da culpa;

Baixa autoestima situacional

- Determinar a confiança do paciente no próprio julgamento;

- Transmitir confiança na capacidade do paciente para lidar com a situação; e

- Ajudar a estabelecer metas realistas para atingir uma autoestima maior.

- Manter registro preciso de ingestão e eliminação;

- Avaliar a localização e extensão do edema, se presente;

- Consultar o médico diante de sinais e sintomas de persistência ou piora de excesso de volume de líquidos;

- Distribuir a ingestão de líquidos ao longo das 24h, conforme apropriado; e

- Monitorar o estado de hidratação (p. ex., mucosas úmidas, pulsos adequados e pressão sanguínea ortostática), conforme apropriado.

- Reconhecer e informar a presença de desequilíbrios eletrolíticos;

- Monitorar a ocorrência do sinal de Chvostek e/ou Trousseau;

- Monitorar ocorrência de manifestação neurológica de desequilíbrio eletrolítico (p. ex., alteração sensorial e fraqueza);

Risco de desequilíbrio eletrolítico

- Observar mudanças na sensibilidade periférica, como dormência e tremores;

- Observar a força muscular;

- Monitorar ocorrência de náusea, vômito e diarreia; e

- Consultar o médico se persistirem ou piorarem os sinais e sintomas de desequilíbrio hídrico e/ ou eletrolítico.

- Reconhecer e informar a presença de desequilíbrios eletrolíticos;

- Monitorar a ocorrência do sinal de Chvostek e/ou Trousseau;

- Monitorar ocorrência de manifestação neurológica de desequilíbrio eletrolítico (p. ex., alteração sensorial e fraqueza);

Risco de volume deficiente de líquidos

- Observar mudanças na sensibilidade periférica, como dormência e tremores;

- Observar a força muscular;

- Monitorar ocorrência de náusea, vômito e diarreia; e

- Consultar o médico se persistirem ou piorarem os sinais e sintomas de desequilíbrio hídrico e/ ou eletrolítico.

Fonte: Dados do estudo, Cacoal, 2017.

\section{DISCUSSÃO}

A SSJ e a NET são dois tipos graves de hipersensibilidade a drogas, caracterizadas por uma extensa separação epidérmica da derme, diferenciando-se apenas em suas extensões. No caso descrito de SSJ-NET, chegaram à conclusão de que o uso do analgésico dipirona tenha sido a causa do desenvolvimento e do agravamento da doença. Tal assertiva também está em consonância com a literatura científica, a qual descreve 100 drogas associadas à etiologia desta afecção, sendo as mais comumente relacionadas: sulfonamidas, anticonvulsivantes, anti-inflamatórios não-hormonais e alopurinol. No entanto, nos 
países em que a dipirona é comercializada, se relaciona frequentemente a essas reações ${ }^{(18)}$.

Algumas pesquisas indicam que sua origem se relacione a uma desregulação da imunidade celular. Sendo a principal contribuinte para tal, a célula $T$ específica, que é a principal mediadora (Células T CD8 + na maioria dos casos, o que difere das demais reações alérgicas da pele, CD4+ e Células NK), por meio da formação de FasL ou perforina/granzima e morte de queratinócitos ${ }^{(19)}$.Tal mecanismo, por meio do Fas ligante (FasL), que liga-se aos queratinócitos via membrana ou Fasl solúvel das células $\mathrm{T}$, atuando na morte dos queratinócitos que causam as reações idiossincráticas a fármacos e a morte celular ${ }^{(1, I I)}$.

Em relação às complicações, a sequela mais frequentemente relatada é o comprometimento ocular, que ocorre em mais da metade dos pacientes com SSJ/NET. No relato de caso descrito acima, o adolescente necessitou de acompanhamento oftalmológico, tendo sido prescrito o colírio Trisorb ( I,0 mg de dextrana 70, 3,0 $\mathrm{mg}$ de hipromelose e 2,0 mg de glicerol) 2/2 horas, para lubrificação. Esse achado se identifica muito com os encontrados em um estudo ${ }^{(17)}$ que, ao analisar 22 pacientes com SSJ, observou que 21 deles apresentaram complicações oftalmológicas, demostrando que é importante a utilização de algum lubrificante para a preservação da superfície ocular.

Com relação ao tratamento utilizado, além dos cuidados gerais e a antibioticoterapia, foi administrada a Imunoglobulina $\mathrm{lg} / \mathrm{kg} / \mathrm{dia}$, apresentando resultados positivos após uma semana, com redução das lesões da mucosa oral, como também das lesões bolhosas disseminadas pelo corpo, marcado pelo aparecimento de crostas e reepitelização da pele. Apesar do benefício observado com a utilização da imunoglobulinoterapia, que é umas das primeiras linhas de tratamento utilizadas, existem controvérsias relacionadas ao tema, em virtude da escassez de informações disponíveis. Sabe-se que a imunoglobulina age na supressão da hipersensibilidade e dificulta a apoptose celular. Além disso, o imunobiológico apresenta uma ação dupla, atuando na redução da internalização das células apresentadoras de antígeno, reduzindo a resposta celular aos antígenos específicos para $T c d 4$, atuando na inibição da ativação das células Tcd8 e os marcadores citotóxicos (perforina); na redução do número de células NK no sangue periférico e da liberação de granzima B no plasma. Como, também, inibe a apoptose de queratinócitos mediada por Faz, por meio de anticorpos que protegem os queratinócitos, bloqueando naturalmente o $\mathrm{Faz}^{(19)}$.

Em relação aos benefícios do uso da imunoglobulina, o estudo constatou que a administração de doses altas (dose total de $2 \mathrm{~g} / \mathrm{kg}$ ) pode reduzir I,78 dias de internação ${ }^{(19)}$. No entanto, em relação à redução de mortalidade, uma grande análise retrospectiva do tratamento com IVIG na SSJ/NET concluiu que a IVIG não conferiu um benefício estatisticamente significativo em comparação à mortalidade prevista por LEE et. al (20/3) ${ }^{(20)}$. Levando em consideração o estudo acima, foi possível chegar à conclusão de que, embora a imunoglobinoterapia seja uma abordagem muito utilizada no tratamento de SSJ/NET, é mais importante priorizar e otimizar os cuidados de suporte e considerar as especificidades de cada paciente antes de utilizá - la, em especial nos extremos das idades ${ }^{(1)}$.

\section{CONCLUSÃO}

A SSJ e a NET são afecções com um considerável poder de morbidade, por isso o seu diagnóstico correto, assim como a aplicação do processo de enfermagem, são importantes para a adequada intervenção clínica. Neste sentido, o processo de enfermagem constitui-se um meio de traçar estratégias que visam estruturar o cuidado ao paciente, sendo uma ferramenta importante no processo de cuidar, proporcionando ao profissional enfermeiro uma organização das informações recebidas do paciente, avaliação, planejamento e interpretação quanto aos resultados, além de possibilitar uma melhor forma de comunicação entre a equipe interdisciplinar.

Dentre as limitações encontradas neste estudo, estão as escassas pesquisas relacionadas a esse agravo. Em sua maioria eram revisões bibliográficas e relatos/estudo de casos de característica biomédica, existindo uma grande deficiência de estudos voltados para área da enfermagem.

Baseado neste fato, este estudo torna-se uma importante fonte do saber para os profissionais da saúde, principalmente na área de enfermagem, a fim de promover mais estudos relacionados, objetivando a melhoria na qualidade da assistência à saúde.

Outro aspecto muito importante evidenciado neste estudo foram as grandes controvérsias quanto à utilização da IVIG no tratamento de pacientes com SSJ/NET, no entanto, neste paciente em questão, houve uma resposta positiva ao tratamento imunomodulador.

Desta forma, concluímos que a enfermagem se constitui parte essencial no processo de recuperação, por isso é preciso que haja um raciocínio clínico que cooperem para decisões assertivas no processo de cuidado, que contribuam para o levantamento dos diagnósticos e planejamento das intervenções necessárias ao reestabelecimento do estado de saúde do cliente. 


\section{REFERÊNCIAS}

I. Schneider JA, Cohen PR. Stevens-Johnson Syndrome and Toxic Epidermal Necrolysis: A Concise Review with a Comprehensive Summary of Therapeutic Interventions Emphasizing Supportive Measures. Adv Ther. [Internet]. 2017;34(6):1235-44. Available from: doi: 10.1007/s 12325$017-0530-y$.

2. Ribeiro AG de A, Ribeiro MC, Benito LAO. Síndrome de Stevens-Johnson (SSJ) em adultos: revisão sistemática. Univ Ciências da Saúde [Internet]. 2018;15(2):II7-25. Disponível em: https://www.publicacoes.uniceub.br/cienciasaude/ article/view/386I

3. Hsu DY, Brieva J, Silverberg NB, Silverberg JI. Morbidity and Mortality of Stevens-Johnson Syndrome and Toxic Epidermal Necrolysis in United States Adults. J Invest Dermatol [Internet]. 20 I6;136(7):I387-97. Disponível em: http://dx. doi.org/10.1016/j.jid.2016.03.023

4. Yang M, Lee JY, Kim J, Kim G, Kim B, Kim J, et al. Incidence of Stevens-Johnson Syndrome and Toxic Epidermal Necrolysis: A Nationwide Population-Based Study Using National Health Insurance Database in Korea. PLoS One. 20I6; II (II):I-I2.

5. Barbosa Emerick MF, Toledo Rodrigues MM, Sampaio Pedrosa DMA, Garbi Novaes MRC, Donato Gottems LB. Síndrome de Stevens-Johnson e Necrólise Epidérmica Tóxica em um hospital do Distrito Federal. Rev Bras Enferm [Internet]. 20|4;67(6):898-904. Available from: http://search.ebscohost.com/login.aspx?direct=true\&db=cin20\&AN $=103880282 \&$ site $=$ ehost-live

6. Frey N, Jossi J, Bodmer M, Bircher A, Jick SS, Meier CR, et al. he Epidemiology of Stevens-Johnson Syndrome and Toxic Epidermal Necrolysis in the UK. J Invest Dermatol [Internet]. 2017;/37(6):I240-7. Available from: http://dx.doi. org/10.1016/j.jid.2017.01.031

7. Xu L, Zhu Y, Yu J, Deng M, Zhu X. Nursing care of a boy seriously infected with Steven-Johnson syndrome after treatment with azithromycin. Med (United States). [Internet]. 2018;97(I):9-12. Available from: http://dx. doi:10.1097/ MD.00000000000091। 2

8. Wang L, Mei XL. Retrospective analysis of stevens-johnson syndrome and toxic epidermal necrolysis in 88 Chinese patients. Chin Med J (Engl). [Internet]. 2017;130(9):1062-8. Available from: http://dx.doi: 10.4103/0366-6999.204929.

9. Bastuji-Garin S, Rzany B SR. Clinical classification of cases of toxic epidermal necrolysis, Stevens-Johnson syndrome, and erythema multiforme. Arch Dermatol. [Internet] 1993;129:92-6. Available from: http://dx.doi:10.1001/archderm.1993.01680220104023

10. Levy AF, Karnikowski M de R e, Campos LCE. Síndrome de Stevens-Johnson e Necrólise Epidérmica Tóxica. ActaMédPort. [Internet] 2014;35(7). Disponível em:http://docs.bvsalud.org/biblioref/2018/04/882866/sindrome-de-stevensjohnson-e-necrolise-epidermica-toxica.pdf
I I. Pimenta MMG. Fisiopatologia e factores de risco genético associados a SSJ/NET. Será possível prevenir as toxicodermias graves? Dissertação - Artigo de Revisão Bibliográfica Mestrado Integrado em Medicina - 2010/I I. Instituto de Ciências Biomédicas Abel Salazar - Universidade do Porto; 2012.

12. Zimmermann S, Sekula P, Venhoff M, Motschall E, Knaus J, Schumacher $M$, et al. Systemic immunomodulating therapies for Stevens-Johnson syndrome and toxic epidermal necrolysis: A systematic review and meta-analysis. JAMA Dermatology. 2017; I53(6):5 I4-22.

13. NANDA. North American Nursing Diagnosis Association. Diagnósticos de enfermagem da NANDA: Definições e classificação 2018-2020. I I th ed.Artmed, editor. Porto Alegre; 2018 . II $87 \mathrm{p}$.

I4. Bulechek GM, City I, Butcher HK, City I, Dochterman JM. Classificação Das Intervenções De Enfermagem (NIC). 5th ed. Guedes R, editor. Rio de Janeiro: Elsevier; 2010. 1037 p.

15. Medic Pejic L, Garcia-Torrent J, Fernandez-Anez N, Lebecki K. Diagnósticos de enfermagem para o paciente com necrólise epidérmica tóxica: estudo de caso. DYNA. 2015;82(I89):72-6.

16. Araújo TM de, Araújo MFM de, Caetano JÁ, Galvão MTG, Damasceno MMC. Diagnósticos de enfermagem para pacientes em risco de desenvolver úlcera por pressão. Rev Bras Enferm [Internet]. 20I I;64(4):67I-6. Available from: http://www.scielo.br/scielo.php?script=sci_arttext\&pi$d=S 00347$ I 6720 I $1000400007 \&$ Ing=pt\&tIng=pt

17. Franca MD, Lima JPG, de Freitas D, Cunha M, Pereira Gomes JA. Estudo dos achados oculares na síndrome de StevensJohnson em pacientes de centro de referência de atendimento terciário. Arq Bras Oftalmol. [Internet]. 2009;72(3):3704. Available from: http://www.scielo.br/scielo.php?script=sci_arttext\&pid=S0004-274920090003000 I 7\&lng=en. https://doi.org/l 0.1590/S0004-274920090003000I7.

18. Souza MCA, Correia APS, Magrani GC, Magrani GC, Côrtes Junior JCS, Côrtes PPR. Síndrome de stevens-johnson e necrólise epidérmica tóxica: relato de caso. Acta Biomed Bras. 2018;9(I): I84-90.

19. Ye LP, Zhang C, Zhu QX. The effect of intravenous immunoglobulin combined with corticosteroid on the progression of stevens-Johnson syndrome and toxic epidermal necrolysis: A meta-Analysis. PLoS One. [Internet]. 2016; I I(I I):I-I7. Available from: https://doi.org/I0.137I/ journal.pone.0167I20

20. Lee HY, Lim YL, Thirumoorthy T PS. The role of intravenous immunoglobulin in toxic epidermal necrolysis: a retrospective analysis of 64 patients managed in a specialized centre. $\mathrm{Br} J$ Dermatol. [Internet]. 2013;169(6):1304-9. Available from: https://doi.org/10.1 I I I/bjd.12607

Recebido: 2019-10-06 Aceito: 2020-04- 15 